\title{
IIIDYT at IEST 2018: Implicit Emotion Classification With Deep Contextualized Word Representations
}

\author{
Jorge A. Balazs, Edison Marrese-Taylor and Yutaka Matsuo \\ Graduate School of Engineering \\ The University of Tokyo \\ \{jorge, emarrese, matsuo\}@weblab.t.u-tokyo.ac.jp
}

\begin{abstract}
In this paper we describe our system designed for the WASSA 2018 Implicit Emotion Shared Task (IEST), which obtained $2^{\text {nd }}$ place out of 30 teams with a test macro F1 score of 0.710. The system is composed of a single pre-trained ELMo layer for encoding words, a Bidirectional Long-Short Memory Network BiLSTM for enriching word representations with context, a max-pooling operation for creating sentence representations from them, and a Dense Layer for projecting the sentence representations into label space. Our official submission was obtained by ensembling 6 of these models initialized with different random seeds. The code for replicating this paper is available at https: / / github.com/ jabalazs/implicit_emotion.
\end{abstract}

\section{Introduction}

Although the definition of emotion is still debated among the scientific community, the automatic identification and understanding of human emotions by machines has long been of interest in computer science. It has usually been assumed that emotions are triggered by the interpretation of a stimulus event according to its meaning.

As language usually reflects the emotional state of an individual, it is natural to study human emotions by understanding how they are reflected in text. We see that many words indeed have affect as a core part of their meaning, for example, dejected and wistful denote some amount of sadness, and are thus associated with sadness. On the other hand, some words are associated with affect even though they do not denote affect. For example, failure and death describe concepts that are usually accompanied by sadness and thus they denote some amount of sadness. In this context, the task of automatically recognizing emotions from text has recently attracted the attention of re- searchers in Natural Language Processing. This task is usually formalized as the classification of words, phrases, or documents into predefined discrete emotion categories or dimensions. Some approaches have aimed at also predicting the degree to which an emotion is expressed in text (Mohammad and Bravo-Marquez, 2017).

In light of this, the WASSA 2018 Implicit Emotion Shared Task (IEST) (Klinger et al., 2018) was proposed to help find ways to automatically learn the link between situations and the emotion they trigger. The task consisted in predicting the emotion of a word excluded from a tweet. Removed words, or trigger-words, included the terms "sad", "happy", "disgusted", "surprised", "angry", "afraid" and their synonyms, and the task was to predict the emotion they conveyed, specifically sadness, joy, disgust, surprise, anger and fear.

From a machine learning perspective, this problem can be seen as sentence classification, in which the goal is to classify a sentence, or in particular a tweet, into one of several categories. In the case of IEST, the problem is specially challenging since tweets contain informal language, the heavy usage of emoji, hashtags and username mentions.

In this paper we describe our system designed for IEST, which obtained the second place out of 30 teams. Our system did not require manual feature engineering and only minimal use of external data. Concretely, our approach is composed of a single pre-trained ELMo layer for encoding words (Peters et al., 2018), a Bidirectional LongShort Memory Network (BiLSTM) (Graves and Schmidhuber, 2005; Graves et al., 2013), for enriching word representations with context, a maxpooling operation for creating sentence representations from said word vectors, and finally a Dense Layer for projecting the sentence representations into label space. To the best of our knowledge, 
our system, which we plan to release, is the first to utilize ELMo for emotion recognition.

\section{Proposed Approach}

\subsection{Preprocessing}

As our model is purely character-based, we performed little data preprocessing. Table 1 shows the special tokens found in the datasets, and how we substituted them.

\begin{tabular}{ll}
\hline Original & Replacement \\
\hline$[\#$ HRIGGERWORD\# ] & --TRIGGERWORD _- \\
@USERNAME & --USERNAME_-- \\
{$[$ NEWLINE] } & --NEWLINE_-- \\
http://url.removed & --URL_- $_{-}$ \\
\hline
\end{tabular}

Table 1: Preprocessing substitutions.

Furthermore, we tokenized the text using a variation of the twokenize.py ${ }^{1}$ script, a Python port of the original Twokenize. java (Gimpel et al., 2011). Concretely, we created an emojiaware version of it by incorporating knowledge from an emoji database, ${ }^{2}$ which we slightly modified for avoiding conflict with emoji sharing unicode codes with common glyphs used in Twitter, ${ }^{3}$ and for making it compatible with Python 3.

\subsection{Architecture}

Figure 1 summarizes our proposed architecture. Our input is based on Embeddings from Language Models (ELMo) by Peters et al. (2018). These are character-based word representations allowing the model to avoid the "unknown token" problem. ELMo uses a set of convolutional neural networks to extract features from character embeddings, and builds word vectors from them. These are then fed to a multi-layer Bidirectional Language Model (BiLM) which returns context-sensitive vectors for each input word.

We used a single-layer BiLSTM as context finetuner (Graves and Schmidhuber, 2005; Graves et al., 2013), on top of the ELMo embeddings, and then aggregated the hidden states it returned by using max-pooling, which has been shown to perform well on sentence classification tasks (Conneau et al., 2017).

\footnotetext{
$\overline{1_{\text {https://github.com/myleott/ark-twokenize-py }}}$

https://github.com/carpedm20/emoji/blob/e7bff32/emoji/ unicode_codes.py

${ }^{3}$ For example, the hashtag emoji is composed by the unicode code points $U+23 \mathrm{U}+\mathrm{FEOF} \quad \mathrm{U}+20 \mathrm{E} 3$, which include $\mathrm{U}+23$, the same code point for the \# glyph.
}

Finally, we used a single-layer fully-connected network for projecting the pooled BiLSTM output into a vector corresponding to the label logits for each predicted class.

\subsection{Implementation Details and Hyperparameters}

ELMo Layer: We used the official AllenNLP implementation of the ELMo model ${ }^{4}$, with the official weights pre-trained on the 1 Billion Word Language Model Benchmark, which contains about $800 \mathrm{M}$ tokens of news crawl data from WMT 2011 (Chelba et al., 2014).

Dimensionalities: By default the ELMo layer outputs a 1024-dimensional vector, which we then feed to a BiLSTM with output size 2048, resulting in a 4096-dimensional vector when concatenating forward and backward directions for each word of the sequence ${ }^{5}$. After max-pooling the BiLSTM output over the sequence dimension, we obtain a single 4096-dimensional vector corresponding to the tweet representation. This representation is finally fed to a single-layer fully-connected network with input size 4096, 512 hidden units, output size 6 , and a ReLU nonlinearity after the hidden layer. The output of the dense layer is a 6-dimensional logit vector for each input example.

Loss Function: As this corresponds to a multiclass classification problem (predicting a single class for each example, with more than 2 classes to choose from), we used the Cross-Entropy Loss as implemented in PyTorch (Paszke et al., 2017).

Optimization: We optimized the model with Adam (Kingma and Ba, 2014), using default hyperparameters $\left(\beta_{1}=0.9, \beta_{2}=0.999, \epsilon=10^{-8}\right)$, following a slanted triangular learning rate schedule (Howard and Ruder, 2018), also with default hyperparameters (cut_frac $=0.1$, ratio $=32$ ), and a maximum learning rate $\eta_{\max }=0.001$, over $T=23,970$ iterations $^{6}$.

Regularization: we used a dropout layer (Srivastava et al., 2014), with probability of 0.5 after both the ELMo and the hidden fully-connected layer, and another one with probability of 0.1 af-

\footnotetext{
4 https://allenai.github.io/allennlp-docs/api/allennlp.

${ }^{5}$ A BiLSTM is composed of two separate LSTMs that read the input in opposite directions and whose outputs are concatenated at the hidden dimension. This results in a vector double the dimension of the input for each time step.

${ }^{6}$ This number is obtained by multiplying the number of epochs (10), times the total number of batches, which for the training dataset corresponds to 2396 batches of 64 elements, and 1 batch of 39 elements, hence $2397 \times 10=23,970$.
} 


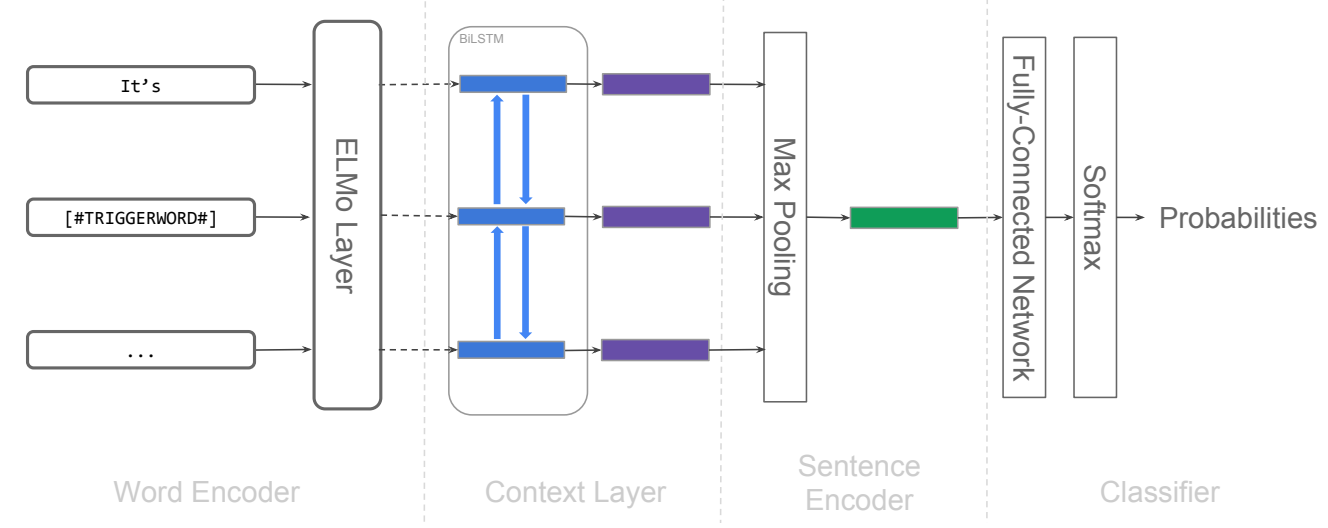

Figure 1: Proposed architecture.

ter the max-pooling aggregation layer. We also reshuffled the training examples between epochs, resulting in a different batch for each iteration.

Model Selection: To choose the best hyperparameter configuration we measured the classification accuracy on the validation (trial) set.

\subsection{Ensembles}

Once we found the best-performing configuration we trained 10 models using different random seeds, and tried averaging the output class probabilities of all their possible $\sum_{k=1}^{9}\left(\begin{array}{l}9 \\ k\end{array}\right)=511$ combinations. As Figure 2 shows, we empirically found that a specific combination of 6 models yielded the best results ( $70.52 \%$ ), providing evidence for the fact that using a number of independent classifiers equal to the number of class labels provides the best results when doing average ensembling (Bonab and Can, 2016).

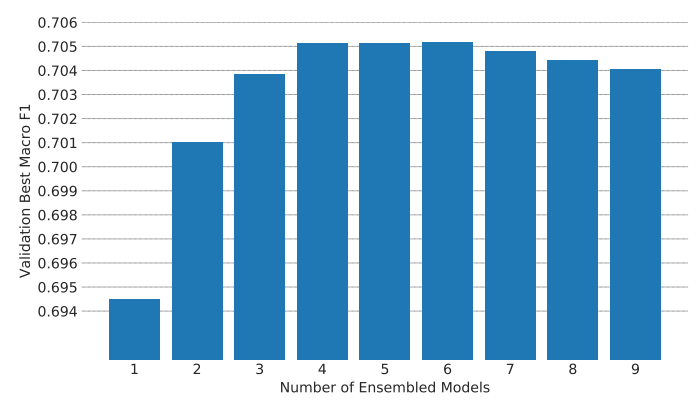

Figure 2: Effect of the number of ensembled models on validation performance.

\section{Experiments and Analyses}

We performed several experiments to gain insights on how the proposed model's performance inter- acts with the shared task's data. We performed an ablation study to see how some of the main hyperparameters affect performance, and an analysis of tweets containing hashtags and emoji to understand how these two types of tokens help the model predict the trigger-word's emotion. We also observed the effects of varying the amount of data used for training the model to evaluate whether it would be worthwhile to gather more training data.

\subsection{Ablation Study}

We performed an ablation study on a single model having obtained $69.23 \%$ accuracy on the validation set. Results are summarized in Table 2.

We can observe that the architectural choice that had the greatest impact on our model was the ELMo layer, providing a $3.71 \%$ boost in performance as compared to using GloVe pre-trained word embeddings.

We can further see that emoji also contributed significantly to the model's performance. In Section 3.4 we give some pointers to understanding why this is so.

Additionally, we tried using the concatenation of the max-pooled, average-pooled and last hidden states of the BiLSTM as the sentence representation, following Howard and Ruder (2018), but found out that this impacted performance negatively. We hypothesize this is due to tweets being too short for needing such a rich representation. Also, the size of the concatenated vector was $4096 \times 3=12,288$, which probably could not be properly exploited by the 512-dimensional fullyconnected layer.

Using a greater BiLSTM hidden size did not help the model, probably because of the reason 


\begin{tabular}{lcc}
\hline Variation & Accuracy $(\boldsymbol{\%})$ & $\boldsymbol{\Delta} \%$ \\
\hline Submitted & $\mathbf{6 9 . 2 3}$ & - \\
\hline No emoji & 68.36 & -0.87 \\
\hline No ELMo & 65.52 & -3.71 \\
\hline Concat Pooling & 68.47 & -0.76 \\
\hline LSTM hidden=4096 & 69.10 & -0.13 \\
LSTM hidden=1024 & 68.93 & -0.30 \\
LSTM hidden=512 & 68.43 & -0.80 \\
\hline POS emb dim $=100$ & 68.99 & -0.24 \\
POS emb dim $=75$ & 68.61 & -0.62 \\
POS emb dim $=50$ & 69.33 & +0.10 \\
POS emb dim=25 & 69.21 & -0.02 \\
\hline SGD optim $1 \mathrm{r}=1$ & 64.33 & -4.90 \\
SGD optim $1 \mathrm{r}=0.1$ & 66.11 & -3.12 \\
SGD optim $1 \mathrm{r}=0.01$ & 60.72 & -8.51 \\
SGD optim $\mathrm{lr}=0.001$ & 30.49 & -38.74 \\
\hline
\end{tabular}

Table 2: Ablation study results.

Accuracies were obtained from the validation dataset. Each model was trained with the same random seed and hyperparameters, save for the one listed. "No emoji" is the same model trained on the training dataset with no emoji, "No ELMo" corresponds to having switched the ELMo word encoding layer with a simple pre-trained GloVe embedding lookup table, and "Concat Pooling" obtained sentence representations by using the pooling method described by Howard and Ruder (2018). "LSTM hidden" corresponds to the hidden dimension of the BiLSTM, "POS emb dim" to the dimension of the part-of-speech embeddings, and "SGD optim lr" to the learning rate used while optimizing with the schedule described by Conneau et al. (2017)

mentioned earlier; the fully-connected layer was not big or deep enough to exploit the additional information. Similarly, using a smaller hidden size neither helped.

We found that using 50-dimensional part-ofspeech embeddings slightly improved results, which implies that better fine-tuning this hyperparameter, or using a better POS tagger could yield an even better performance.

Regarding optimization strategies, we also tried using SGD with different learning rates and a stepwise learning rate schedule as described by Conneau et al. (2018), but we found that doing this did not improve performance.

Finally, Figure 3 shows the effect of using different dropout probabilities. We can see that having higher dropout after the word-representation layer and the fully-connected network's hidden layer, while having a low dropout after the sentence encoding layer yielded better results overall.

\subsection{Error Analysis}

Figure 4 shows the confusion matrix of a single model evaluated on the test set, and Table 3 the

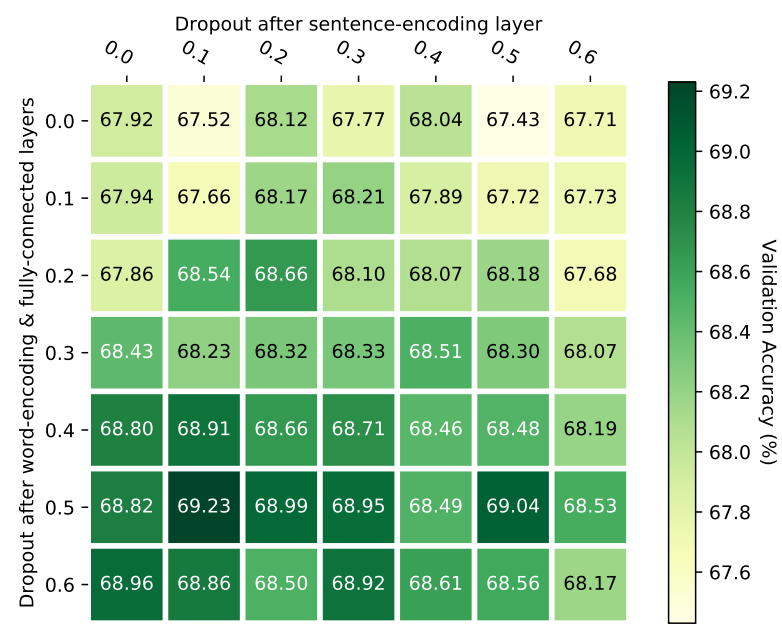

Figure 3: Dropout Ablation.

Rows correspond to the dropout applied both after the ELMo layer (word encoding layer) and after the fully-connected network's hidden layer, while columns correspond to the dropout applied after the max-pooling operation (sentence encoding layer.)

corresponding classification report. In general, we confirm what Klinger et al. (2018) report: anger was the most difficult class to predict, followed by surprise, whereas joy, fear, and disgust are the better performing ones.

To observe whether any particular pattern arose from the sentence representations encoded by our model, we projected them into $3 \mathrm{~d}$ space through Principal Component Analysis (PCA), and were surprised to find that 2 clearly defined clusters emerged (see Figure 6), one containing the majority of datapoints, and another containing joy tweets exclusively. Upon further exploration we also found that the smaller cluster was composed only by tweets containing the pattern un _-TRIGGERWORD_-, and further, that all of them were correctly classified.

It is also worth mentioning that there are 5827 tweets in the training set with this pattern. Of these, $5822(99.9 \%)$ correspond to the label joy. We observe a similar trend on the test set; 1115 of the 1116 tweets having the un _-TRIGGERWORD _- pattern correspond to joy tweets. We hypothesize this is the reason why the model learned this pattern as a strong discriminating feature.

Finally, the only tweet in the test set that contained this pattern and did not belong to the joy class, originally had unsurprised as its triggerword $^{7}$, and unsurprisingly, was misclassified.

\footnotetext{
${ }^{7}$ We manually searched for the original tweet.
} 


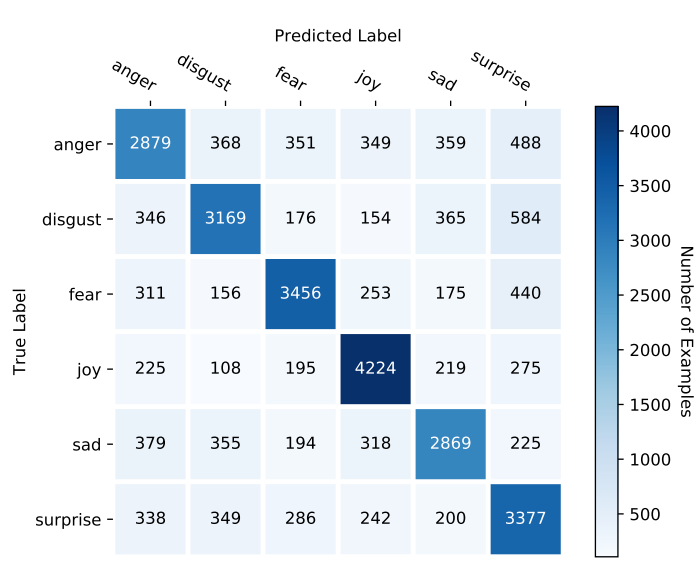

Figure 4: Confusion Matrix (Test Set).

\begin{tabular}{cccc}
\hline & Precision & Recall & F1-score \\
\hline anger & 0.643 & 0.601 & 0.621 \\
disgust & 0.703 & 0.661 & 0.682 \\
fear & 0.742 & 0.721 & 0.732 \\
joy & 0.762 & 0.805 & 0.783 \\
sad & 0.685 & 0.661 & 0.673 \\
surprise & 0.627 & 0.705 & 0.663 \\
\hline Average & 0.695 & 0.695 & 0.694 \\
\hline
\end{tabular}

Table 3: Classification Report (Test Set).

\subsection{Effect of the Amount of Training Data}

As Figure 5 shows, increasing the amount of data with which our model was trained consistently increased validation accuracy and validation macro F1 score. The trend suggests that the proposed model is expressive enough to learn from more data, and is not overfitting the training set.

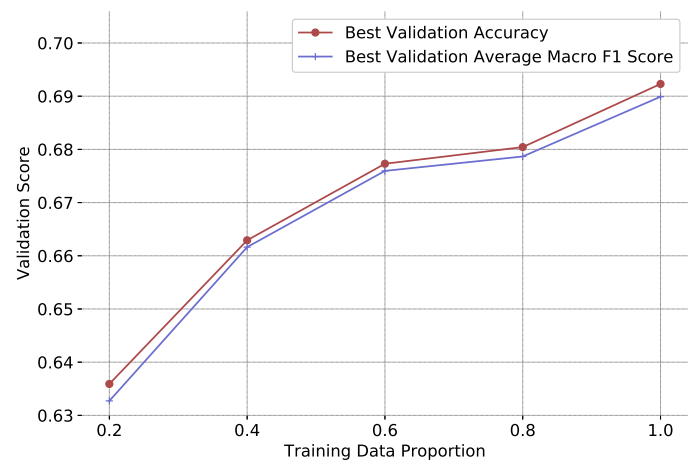

Figure 5: Effect of the amount of training data on classification performance.

\subsection{Effect of Emoji and Hashtags}

Table 4 shows the overall effect of hashtags and emoji on classification performance. Tweets con-

\begin{tabular}{lcc}
\hline & Present & Not Present \\
\hline Emoji & $4805(76.6 \%)$ & $23952(68.0 \%)$ \\
Hashtags & $2122(70.5 \%)$ & $26635(69.4 \%)$ \\
\hline
\end{tabular}

Table 4: Number of tweets on the test set with and without emoji and hashtags. The number between parentheses is the proportion of tweets classified correctly.

taining emoji seem to be easier for the model to classify than those without. Hashtags also have a positive effect on classification performance, however it is less significant. This implies that emoji, and hashtags in a smaller degree, provide tweets with a context richer in sentiment information, allowing the model to better guess the emotion of the trigger-word.

\begin{tabular}{lrrrrrrr}
\hline \multirow{2}{*}{ Emoji alias } & \multirow{2}{*}{$\mathbf{N}$} & \multicolumn{2}{c}{ emoji } & & \multicolumn{2}{c}{ no-emoji } & \multirow{2}{*}{$\boldsymbol{N}$} \\
& & \multicolumn{1}{c}{$\#$} & $\%$ & & $\#$ & $\%$ & \\
\hline mask & 163 & 154 & 94.48 & & 134 & 82.21 & -12.27 \\
two_hearts & 87 & 81 & 93.10 & & 77 & 88.51 & -4.59 \\
heart_eyes & 122 & 109 & 89.34 & & 103 & 84.43 & -4.91 \\
heart & 267 & 237 & 88.76 & & 235 & 88.01 & -0.75 \\
\hline rage & 92 & 78 & 84.78 & & 66 & 71.74 & -13.04 \\
cry & 116 & 97 & 83.62 & & 83 & 71.55 & -12.07 \\
sob & 490 & 363 & 74.08 & & 345 & 70.41 & -3.67 \\
unamused & 167 & 121 & 72.46 & & 116 & 69.46 & -3.00 \\
\hline weary & 204 & 140 & 68.63 & & 139 & 68.14 & -0.49 \\
joy & 978 & 649 & 66.36 & & 629 & 64.31 & -2.05 \\
sweat_smile & 111 & 73 & 65.77 & & 75 & 67.57 & 1.80 \\
confused & 77 & 46 & 59.74 & & 48 & 62.34 & 2.60 \\
\hline
\end{tabular}

Table 5: Fine grained performance on tweets containing emoji, and the effect of removing them.

$\mathbf{N}$ is the total number of tweets containing the listed emoji, $\#$ and $\%$ the number and percentage of correctly-classified tweets respectively, and $\Delta \boldsymbol{\%}$ the variation of test accuracy when removing the emoji from the tweets.

Table 5 shows the effect specific emoji have on classification performance. It is clear some emoji strongly contribute to improving prediction quality. The most interesting ones are mask, rage, and cry, which significantly increase accuracy. Further, contrary to intuition, the sob emoji contributes less than cry, despite representing a stronger emotion. This is probably due to sob being used for depicting a wider spectrum of emotions.

Finally, not all emoji are beneficial for this task. When removing sweat_smile and confused accuracy increased, probably because they represent emotions other than the ones being predicted. 


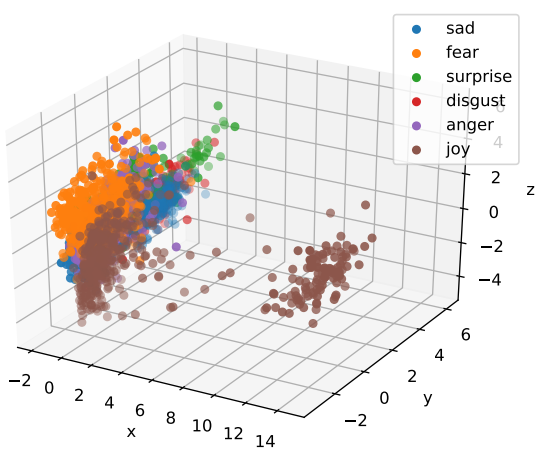

Figure 6: 3d Projection of the Test Sentence Representations.

\section{Conclusions and Future Work}

We described the model that got second place in the WASSA 2018 Implicit Emotion Shared Task. Despite its simplicity, and low amount of dependencies on libraries and external features, it performed almost as well as the system that obtained the first place.

Our ablation study revealed that our hyperparameters were indeed quite well-tuned for the task, which agrees with the good results obtained in the official submission. However, the ablation study also showed that increased performance can be obtained by incorporating POS embeddings as additional inputs. Further experiments are required to accurately measure the impact that this additional input may have on the results. We also think the performance can be boosted by making the architecture more complex, concretely, by using a BiLSTM with multiple layers and skip connections in a way akin to (Peters et al., 2018), or by making the fully-connected network bigger and deeper.

We also showed that, what was probably an annotation artifact, the un _- TRIGGERWORD _pattern, resulted in increased performance for the joy label. This pattern was probably originated by a heuristic naïvely replacing the ocurrence of happy by the trigger-word indicator. We think the dataset could be improved by replacing the word unhappy, in the original examples, by _-_RRIGGERWORD__ instead of un _- TRIGGERWORD _-, and labeling it as sad, or angry, instead of joy.

Finally, our studies regarding the importance of hashtags and emoji in the classification showed that both of them seem to contribute significantly to the performance, although in different measures.

\section{Acknowledgements}

We thank the anonymous reviewers for their reviews and suggestions. The first author is partially supported by the Japanese Government MEXT Scholarship.

\section{References}

Hamed R. Bonab and Fazli Can. 2016. A theoretical framework on the ideal number of classifiers for online ensembles in data streams. In Proceedings of the 25th ACM International on Conference on Information and Knowledge Management, CIKM '16, pages 2053-2056, New York, NY, USA. ACM.

Ciprian Chelba, Tomas Mikolov, Mike Schuster, Qi Ge, Thorsten Brants, Phillipp Koehn, and Tony Robinson. 2014. One billion word benchmark for measuring progress in statistical language modeling. Proceedings of the Annual Conference of the International Speech Communication Association, INTERSPEECH, pages 2635-2639.

Alexis Conneau, Douwe Kiela, Holger Schwenk, Loï Barrault, and Antoine Bordes. 2017. Supervised Learning of Universal Sentence Representations from Natural Language Inference Data. In Proceedings of the 2017 Conference on Empirical Methods in Natural Language Processing, pages 670-680, Copenhagen, Denmark. Association for Computational Linguistics.

Alexis Conneau, Germán Kruszewski, Guillaume Lample, Loïc Barrault, and Marco Baroni. 2018. What you can cram into a single vector: Probing sentence embeddings for linguistic properties. In Proceedings of the 56th Annual Meeting of the Association for Computational Linguistics (Volume 1: Long Papers), pages 2126-2136. Association for Computational Linguistics.

Kevin Gimpel, Nathan Schneider, Brendan O'Connor, Dipanjan Das, Daniel Mills, Jacob Eisenstein, Michael Heilman, Dani Yogatama, Jeffrey Flanigan, and Noah A. Smith. 2011. Part-of-speech tagging for twitter: Annotation, features, and experiments. In Proceedings of the 49th Annual Meeting of the Association for Computational Linguistics: Human Language Technologies, pages 42-47, Portland, Oregon, USA. Association for Computational Linguistics.

Alex Graves, Abdel-rahman Mohamed, and Geoffrey Hinton. 2013. Speech Recognition with Deep Recurrent Neural Networks. In Proceedings of the 2013 International Conference on Acoustics, Speech and Signal Processing (ICASSP), pages 6645-6649, Vancouver, Canada. IEEE. 
Alex Graves and Jürgen Schmidhuber. 2005. Framewise Phoneme Classification with Bidirectional LSTM and Other Neural Network Architectures. Neural Networks, 18(5-6):602-610.

Jeremy Howard and Sebastian Ruder. 2018. Universal Language Model Fine-tuning for Text Classification. ArXiv e-prints.

Diederik P. Kingma and Jimmy Ba. 2014. Adam: A method for stochastic optimization. CoRR.

Roman Klinger, Orphée de Clercq, Saif M. Mohammad, and Alexandra Balahur. 2018. IEST: WASSA2018 Implicit Emotions Shared Task. In Proceedings of the 9th Workshop on Computational Approaches to Subjectivity, Sentiment and Social Media Analysis, Brussels, Belgium. Association for Computational Linguistics.

Saif M. Mohammad and Felipe Bravo-Marquez. 2017. WASSA-2017 Shared Task on Emotion Intensity. In Proceedings of the EMNLP 2017 Workshop on Computational Approaches to Subjectivity, Sentiment, and Social Media (WASSA), Copenhagen, Denmark.

Adam Paszke, Sam Gross, Soumith Chintala, Gregory Chanan, Edward Yang, Zachary DeVito, Zeming Lin, Alban Desmaison, Luca Antiga, and Adam Lerer. 2017. Automatic differentiation in pytorch. In NIPS Autodiff Workshop.

Matthew Peters, Mark Neumann, Mohit Iyyer, Matt Gardner, Christopher Clark, Kenton Lee, and Luke Zettlemoyer. 2018. Deep contextualized word representations. In Proceedings of the 2018 Conference of the North American Chapter of the Association for Computational Linguistics: Human Language Technologies, Volume 1 (Long Papers), pages 2227-2237, New Orleans, Louisiana. Association for Computational Linguistics.

Nitish Srivastava, Geoffrey Hinton, Alex Krizhevsky, Ilya Sutskever, and Ruslan Salakhutdinov. 2014. Dropout: A simple way to prevent neural networks from overfitting. Journal of Machine Learning Research, 15:1929-1958. 